\title{
Surface and grain boundary complexions in transition metal - bismuth alloys
}

\author{
Qin Gao and Michael Widom \\ Department of Physics, Carnegie Mellon University, Pittsburgh, Pennsylvania 15213, USA
}

\begin{abstract}
This article reviews experimental and theoretical work related to grain boundary complexions in transition metals, especially bismuth alloy complexions on nickel and copper surfaces and grain boundaries. One-, three-, five-, and seven-layer bismuth complexions are observed on the $\mathrm{Ni}(111)$ surface. Recent experiments suggest that Bi impurities segregate to form bilayer complexions on $\mathrm{Ni}$ and $\mathrm{Cu}$ grain boundaries which could possibly explain liquid metal embrittlement. Density functional theory calculations of Bi films on transition metal grain boundaries confirm that Bi bilayer complexions (actually a pair of monolayers bound to the metal surfaces) are thermodynamically stable. Meanwhile, complexion transitions have been demonstrated with molecular dynamics and Monte Carlo simulations and are supported by analytical thermodynamic models.
\end{abstract}

\section{Introduction}

Bismuth replaces lead in solder alloys due to its low melting tempertature and non-toxicity [1]. Bismuth naphthenate is used as environmentally friendly lubricant additives in extreme pressure applications [2]. Moreover, liquid $\mathrm{Bi}$ is used as a spallation target to generate neutrons due to its high neutron production rate [3]. However, liquid $\mathrm{Bi}$ is known to embrittle its solid container through grain boundary (GB) penetration. To understand and utimately solve the liquid metal embrittlement problem, extensive theoretical and experimental studies were performed on Bi at metal GBs. The Micron-scale Bi penetration tips, nanometer-scale integranular films and bilayer complexions were discovered in experiments with $\mathrm{Ni}$ and $\mathrm{Cu}[4,5,6]$.

Analytical thermodynamic models based on ideal solution [7] and critical wetting theory $[8,9,10]$ explain the complexion wetting and prewetting transitions [11]. First principles studies with density functional theory (DFT) calculations confirmed the stability of bilayer films and explained the difference between transition metals [12, 13]. Molecular dynamics (MD) simulation demonstrates complexion transitions in pure $\mathrm{Cu}$ [14] and Ag segre- gated $\mathrm{Cu}$ [15], and agreed well with the diffusion experiments [16]. People debate over whether liquid metal embrittlement (LME) is due to an electronic effect [17] or atomic size effect [18] based on monolayer segregation models.

This review will first cover Bi surface complexions and then concentrate on $\mathrm{Bi}$ complexions at transition metal (TM) GBs from the aspects of experiments, DFT calculations, analytical models and MD simulations. Some perspectives about future directions will also be discussed.

\section{Surface complexion}

Bulk Bi takes the rhombohedral structure of Pearson type hR2 (prototype $\alpha$-As) common to group-V semimetals, which is distorted from the simple cubic structure by a Jones-Peierls mechanism [19]. The bulk Bi structure is best described as a stacking of bilayers in the [001] direction [20] (3-index hexagonal indexing). Three strong covalent bonds of length $3.06 \AA$ link each Bi atom to others within each bilayer, while three weak metallic bonds of length $3.51 \AA$ connect each Bi atom to others in the adjacent bilayer. The (001) plane is thus a natural cleavage 
plane, with divisions expected between bilayers. Additional high pressure forms are also known.

$\mathrm{Bi}$ forms various monolayer structures on $\mathrm{Ni}$ and $\mathrm{Cu}$ surfaces [21, 22, 23, 24, 25, 26, 27, 28]. In multilayer surface complexions $(\sim 1 \mathrm{~nm})$, Bi exhibits allotropic transformations from puckered pseudocubic films to bulk-like rhombohedral films on $\mathrm{Si}(111)$ and on some quasicrystal surfaces [29, 30]. Experiments and first-principles calculations concur that both film types exhibit bilayer growth due to the exotic bonding character of $\mathrm{Bi}$, rather than freeelectron like quantum size effect [31]. In contrast, the initial growth of Bi films on metallic substrates has not been well studied until a recent experiment [32] reported the formation of Bi-Ni surface alloy, and one-, three-, fiveand seven-layer $\mathrm{Bi}$ hexagonal films of $\mathrm{Bi}$ on the $\mathrm{Ni}(111)$ surface. The authors proposed atomic structures and explained their stabilities based on free-electron-like quantum size effect [32].

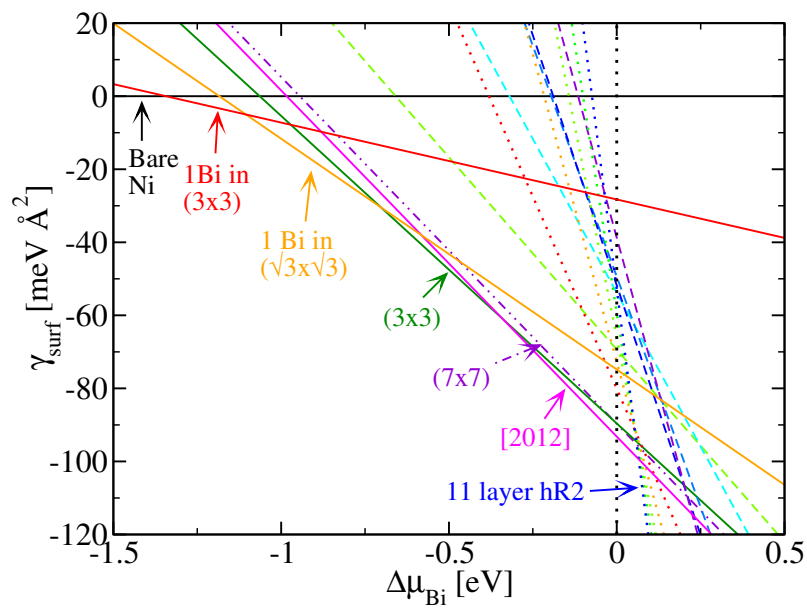

Figure 1: (Color online) Surface free energy of $\mathrm{Bi}$ on (or in the surface layer of) $\mathrm{Ni}(111)$ surface [20]. The black solid line is bare $\mathrm{Ni}(111)$ surface and other solid lines are stable monolayer structures. The dotdashed line is the $(7 \times 7)$ structure proposed in [32], which is predicted not stable. Black dotted line stands for infinite bulk-like Bi films. Other dotted lines are Bi bulk-like (001) films on Ni(111). Dashed lines are the hexagonal films proposed in [32].

DFT calculations were performed within the PBE generalized gradient approximation [33] to verify the stability of the proposed structures and its mechanism [20]. Consider a slab consisting of several layers of elemental $\mathrm{Ni}$ in its FCC structure, with two free surfaces each of area
$A$. Define the energy of this structure as $E_{\mathrm{Ni}}^{\mathrm{Slab}}$. Now place $N_{\mathrm{Bi}}$ atoms on one surface of this slab resulting in a total energy $E_{\mathrm{Tot}}$. The formation enthalpy of $\mathrm{Bi}$ on the surface, relative to the bare slab and bulk bismuth, is

$$
\Delta H=E_{\mathrm{Tot}}-E_{\mathrm{Ni}}^{\mathrm{Slab}}-E_{\mathrm{Bi}}^{\mathrm{Bulk}} N_{\mathrm{Bi}} .
$$

The surface free energy per area, $\gamma_{\text {surf }}$ (shown in Fig. 1), is defined as

$$
\gamma_{\text {surf }}=\Delta H / A \gamma_{\text {surf }}=\left(\Delta H-\Delta \mu_{\mathrm{Bi}} N_{\mathrm{Bi}}\right) / A .
$$

Here we choose to measure the chemical potential of $\mathrm{Bi}$ relative to its bulk value $\Delta \mu_{\mathrm{Bi}}=\mu_{\mathrm{Bi}}-\mu_{\mathrm{Bi}}^{\mathrm{Bulk}}$. Finally, if we replace $\delta N_{\mathrm{Ni}}$ atoms in the slab with Bi atoms (most likely on the outer layer of the slab), then we must replace $E_{\mathrm{Ni}}^{\mathrm{Slab}}$ with $E_{\mathrm{Ni}}^{\mathrm{Slab}}-\delta N_{\mathrm{Ni}} E_{\mathrm{Ni}}^{\mathrm{Bulk}}$. Stable structures minimize $\gamma_{\text {surf }}$ at certain $\Delta \mu_{\mathrm{Bi}}$.

Fig. 1 suggests the stable sequence: a bare $\mathrm{Ni}(111)$ transforms to a dilute surface alloy $(1 \mathrm{Bi}$ in $(3 \times 3)$ cell $)$ at $\Delta \mu_{\mathrm{Bi}}=-1.34 \mathrm{eV}$, the observed $\sqrt{3} \times \sqrt{3}$ surface alloy [32] at $\Delta \mu_{\mathrm{Bi}}=-1.10 \mathrm{eV}$, the observed $(3 \times 3)$ monolayer [32] at $\Delta \mu_{\mathrm{Bi}}=-0.70 \mathrm{eV}$, a [2012] monolayer like that observed in $\mathrm{Bi}$ on $\mathrm{Cu}(111)$ [24] at $\Delta \mu_{\mathrm{Bi}}=-0.34 \mathrm{eV}$, an infinite-height bulk-like hR2 film. DFT study thus found a surface monolayer strongly interacting with $\mathrm{Ni}$, ultimately followed by a bulk-like (001) bilayer films as illustrated in Fig. 2 [20] at $\Delta \mu_{\mathrm{Bi}}=+0.06 \mathrm{eV}$ (this is slightly positive rather than zero owing to the energy cost of the Ni-Bi interface). These structures are much more stable than the previously proposed hexagonal films and are due to the exotic bonding character of $\mathrm{Bi}$ rather than free-electron like quantum size effect.

Formation of bulk $\mathrm{Bi}$ on the Ni surface is of course a nonequilibrium structure created by deposition of $\mathrm{Bi}$ on the Ni surface. In thermodynamic equilibrium bulk Ni-Bi compounds can form at intermediate $\Delta \mu_{\mathrm{Bi}}$. DFT calculations of the bulk phase diagram reveal coexistence of FCC $\mathrm{Ni}$ with monoclinic $\mathrm{BiNi}$ at $\mu_{\mathrm{Bi}}-\mu_{\mathrm{Ni}}=-0.07 \mathrm{eV}$. Assuming $\mu_{\mathrm{Ni}}=0$ in the Ni-rich limit of equilibrium with bulk $\mathrm{Ni}$, we see that formation of this compound occurs in equilibrium after the [2012] monolayer but, of course, before growth of bulk Bi.

Bi surface complexions also exhibit exotic electronic properties. Bi bulk-like hR2 bilayers have been shown to act as two-dimensional topological insulators [34, 35]. A $\mathrm{Bi}(20 \mathrm{~nm}) / \mathrm{Ni}(3 \mathrm{~nm})$ film has been demonstrated to be a p-Wave superconductor [36]. 


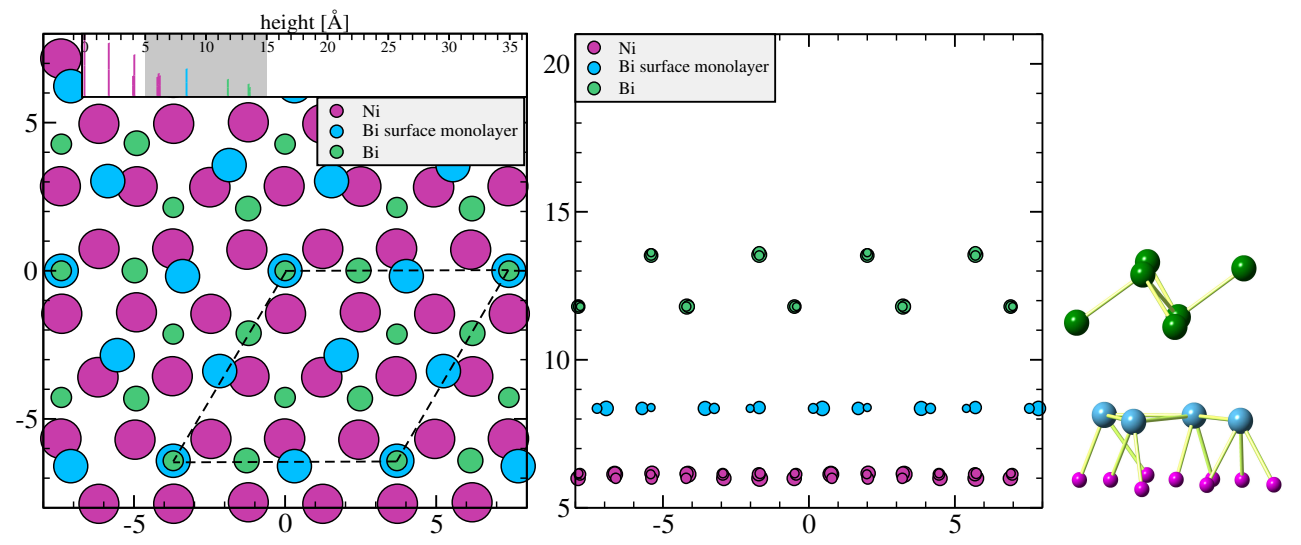

Figure 2: (Color online) Top view (left) and side view (middle) of DFT relaxed three-layer hR2 Bi film on Ni(111) (3×3) cell (dashed). Insert at top of the left-hand figure is a histogram of atoms at different heights. Shading in insert conveys the region illustrated containing the top layer of $\mathrm{Ni}$, the Bi surface monolayer, and additional Bi layers. Atom size indicates depth (large below small). Length units are in $\AA$. Chemical bonding is shown at right (slightly tilted). Adapted from [20].

\section{Grain boundary complexion}

\subsection{Experiments}

Liquid metal embrittlement (LME) is a long standing puzzle for physics and material science community. Severe embrittlement occurs when ductile metals like $\mathrm{Ni}$ and $\mathrm{Cu}$ contact liquid metals like Bi. Experimental studies using Auger Electron Spectroscopy (AES) and scanning electron microscopy (SEM) focused on the properties of the liquid metal penetration tip, which is tens or hundreds of microns long and several microns thick liquid Bi formed by wetting [4] the transition metal GB as shown in Fig. 3(A). The penetration tip dissociates the GB by forming two solid-liquid interfaces which are regarded as complexions [11], with bulk liquid Bi-Ni alloy in-between. The region in front of the penetration tip is believed to be a plastic deformation zone $[39,40]$. Recent experiments found $\mathrm{Mn}, \mathrm{Sn}$, and $\mathrm{Fe}$ impurities in solid Ni enhance the integranular penetration 10 - 20 fold [41]. Based on thermodynamic models, it is inferred that $\mathrm{Mn}$ and Sn segregate to the Ni GB and change the penetration morphologies. While $\mathrm{Fe}$ in solid $\mathrm{Ni}$ is expected to have no effect due to its large solubility, $\mathrm{Fe}$ in a liquid Bi-Ni alloy precipitates, consumes $\mathrm{Ni}$, causes dissolvation of $\mathrm{Ni}$ from the Ni GB, and thus enhance the integranular penetration.

Besides the penetration tip, SEM and AES measurements indicate a nanometer-thick quasi-liquid $\mathrm{Bi}$ inter- granular film (IGF) extends hundreds micrometers ahead the penetration tip when $\mathrm{Ni}$ is in contact with liquid $\mathrm{Bi}$ $\mathrm{Ni}$ alloy [42], extending the crack depth to several times the length of the penetration tip in bending or tensile tests $[39,5]$ as shown in Fig. 3(A, B). By observing an abrupt change in GB grooving angle [43] and in the temperature dependence of the Bi adsorption [44], people inferred the existence of complexion prewetting transition as shown in Fig. 3(C). Moreover, a Bi monolayer complexion on a special $\mathrm{Cu}$ GB was observed [17].

Recently, using aberration-corrected high-angle annular dark-field (HAADF) scanning transmission electron microscopy (STEM), Bi (sub)nanometer thick complexions were directly observed at $\mathrm{Ni}$ general grain boundaries [6], as shown in Fig. 4(A - D). The Bi complexions are mostly bilayers and are ubiquitous at $\mathrm{Ni}$ high energy GBs around the penetration tip. Besides the bilayer complexion, a trilayer complexion coexists with a bilayer complexion as shown in Fig. 4(E), on a single GB near the penetration tip indicates complexion prewetting transition. The two layers in the Bi bilayer complexions are coherent with the Ni GB planes, but not coherent with each other and have large layer spacing(3.9 $\AA$, Fig. 4(F)), which indicates strong $\mathrm{Bi}-\mathrm{Ni}$ interaction and weak $\mathrm{Bi}-\mathrm{Bi}$ interlayer interaction. The weak $\mathrm{Bi}-\mathrm{Bi}$ interlayer interaction could significantly reduce GB cohesion thus lead to embrittlement. The penetration tip and IGF together 


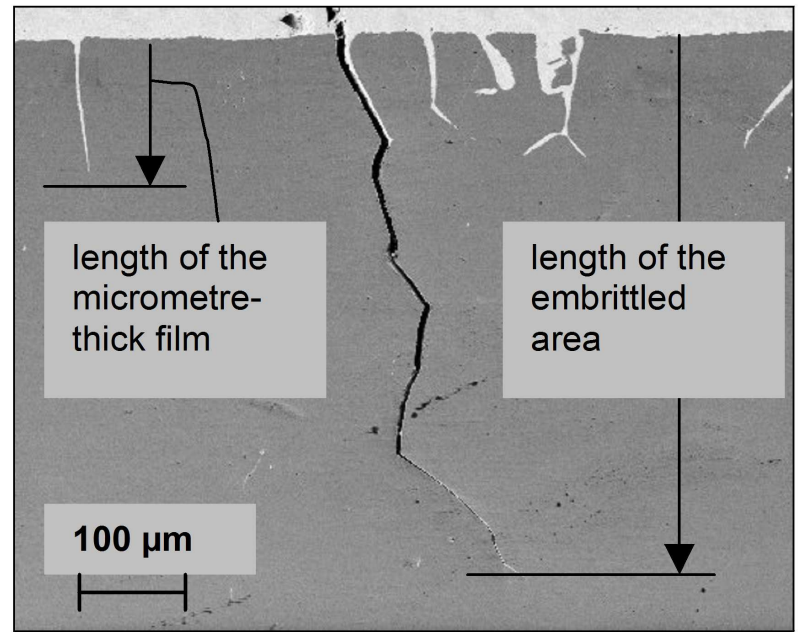

(A)

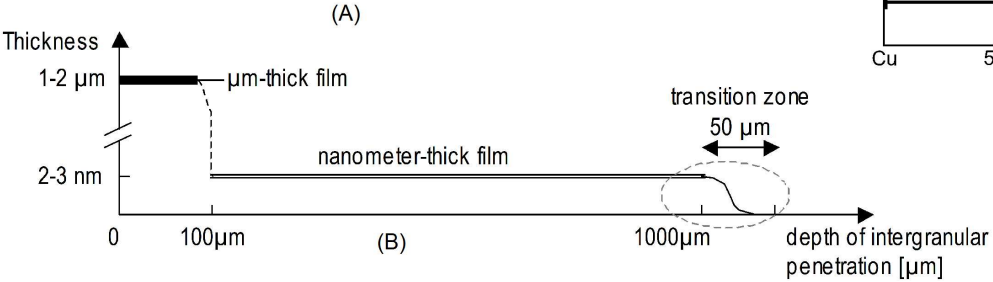

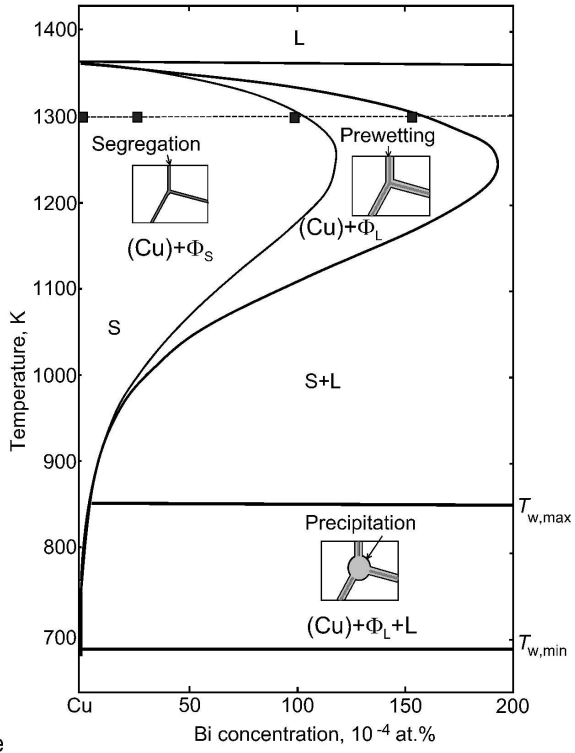

(C)

Figure 3: (A) Cross-section of a Ni polycrystal in contact with liquid Ni-Bi alloy. Top left region shows a micrometer thick intergranular penetration tip. Middle region shows long crack due to the penetration tip and the nanometrer-thick film. (B) Schematic representation of Bi concentration profile across the fracture surface between the external surface and the ductile core in $\mathrm{Ni}-\mathrm{Bi}$ system at $700^{\circ} \mathrm{C}$. $(\mathrm{C}) \mathrm{Cu}-$ rich side of the $\mathrm{Cu}-\mathrm{Bi}$ phase diagram. The thick curve is the (retrograde) bulk solidus line [37]. The thin retrograde curve is the GB solidus line obtained for the Cu-Bi polycrystals [37]. The horizontal lines at $T_{\mathrm{w}, \max }$ and $T_{\mathrm{w}, \mathrm{min}}$ are GB wetting phase transition tie-lines. (A) and (B) are reprinted from [5] with permission, (C) is reprinted from [38] with permission.

with the Bi bilayer complexion, could thus explain the LME. Moreover, similar Bi bilayer complexions were observed on $\mathrm{Cu}$ general GBs [45]. Besides forming bilayer complexions at GBs, Bi also alters the structure of triple junctions due to the strong reduction of GB energies by segregation $[46,47]$.

\subsection{Analytic thermodynamic models}

Analytical thermodynamics models of segregation can explain and predict the complexion transitions. Based on the assumption of monolayer adsorption on an interface with homogenous adsorption sites, the Langmuir-McLean isotherm [50] shows

$$
\frac{X^{s}}{1-X^{s}}=\frac{X}{1-X} e^{-\frac{\Delta H_{s e g}}{R T}},
$$

where $X^{s}$ and $X$ are the atomic fraction of segregants at the GB and in the bulk respectively. $\Delta H_{\text {seg }}$ is the molar enthalpy of segregation, $R$ is the universal gas constant, and $\mathrm{T}$ is temperature. The model generalizes to a multi-layer GB segregation model $[51,52,53]$, where $X^{i}$, the atomic fraction of segregates in the $i^{\text {th }}$ atomic layer from GB replaces $X^{s}$, and the enthalpy of segregation to the $i^{\text {th }}$ layer $\Delta H_{\text {seg }}^{i}$ replaces $\Delta H_{\text {seg. }}$. Wynblatt and $\mathrm{Ku}$ recognized that $\Delta H_{\text {seg }}^{i}$ consists of a chemical contribution [54] which could be derived with bond energy [48] and an elastic contribution that derives from a continuum linear elastic formalism [55]. The model-predicted GB composition profiles [48] agree well with Monte Carlo (MC) simulation [49] as shown in Fig. 5(A). The model 

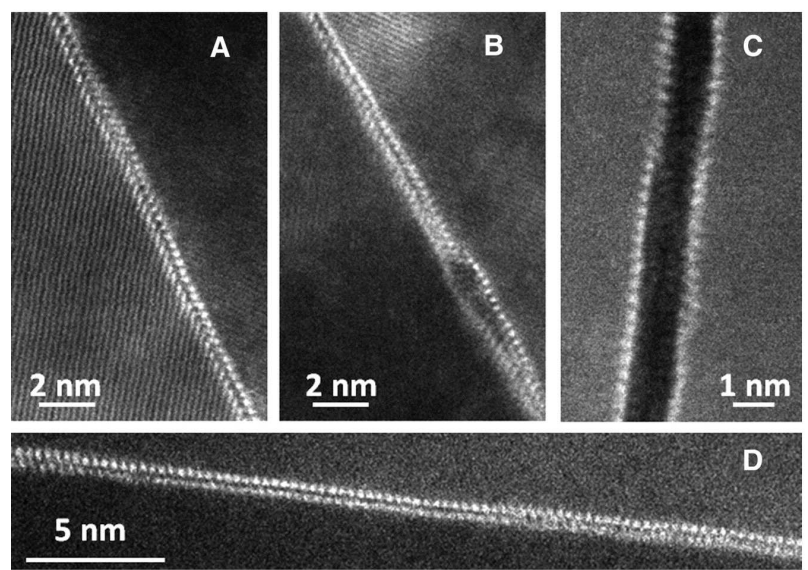
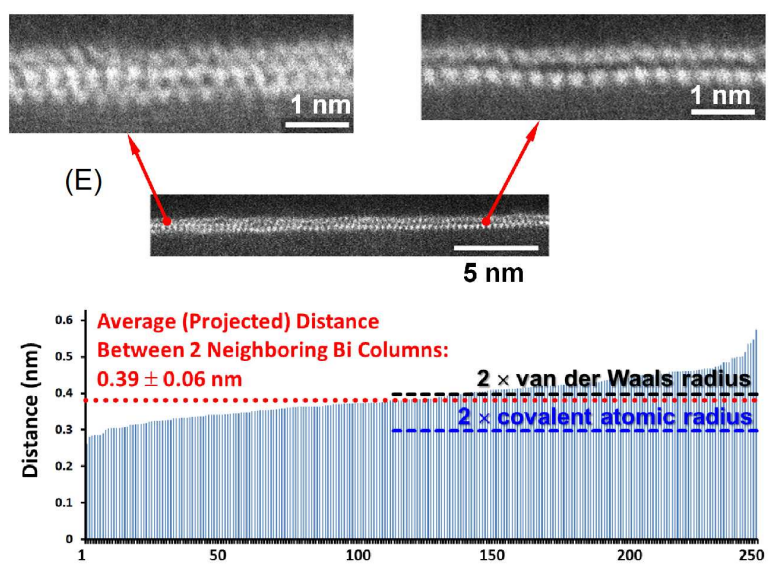

(F) 250 Measurements; Each Bar Represents One Measurement

Figure 4: (Color online)(A, D) STEM HAADF micrographs showing Bi bilayer complexion. (B, C) Brittle decohesion occurs due to the bilayer complexion. (E) Coexistence of bilayer and trilayer interfacial phases at a single GB, indicating a possible GB phase transition between them. (F) Measured (projected) distances between two neighboring adsorbed Bi atom columns across two adsorbed layers. The large layer spacing (3.9 $\AA$ ) between Bi layers indicates weak interaction and embrittlement. All panels are reprinted from [6] with permission from AAAS.

is capable of producing a phase diagram containing wetting and prewetting complexion transitions as shown in Fig. 5(B) [7]. GB layering transitions in bicrystals were studied in [56].

Another approach originates from Cahn's critical point wetting theory [8] which is a phase field model of a binary liquid with a miscibility gap. The model is extended to the binary solid interface $[9,10,57]$ where the excess free energy includes: a linear term in misorientation angle, the squared gradient of composition, the square gradient of crystallinity (characterizing the local structural order), and the excess free energy of a material with no misorientation. This extended model can produce GB phase diagrams containing complexion wetting and prewetting transitions in a binary system with a eutectic transition [58]. Multilayer adsorption was also encorporated in later models [59].

\subsection{Density functional theory calculations}

Density functional theory is helpful to identify the stability of Bi films at GBs. From equilibrium thermodynamics, the most stable structure at a certain Bi chemical potential minimizes the GB free energy $\gamma$, which is similar to $\gamma_{\text {surf }}$ in Eq. 2 but replaces the Ni slab with free surfaces with a TM slab containing GB's. Bi is then introduced to one GB and the GB free energy is calculated as before.

A recent theoretical study [12] of $\mathrm{Bi}$ at $\mathrm{Ni}$ and $\mathrm{Cu}$ found the Bi bilayer is stable on high energy $\Sigma 5(310) \mathrm{GB}$, while on low energy (111) twist GBs the Bi bilayer is unstable. The authors proposed that bilayers are more stable than monolayers based on the interaction strength between $\mathrm{Bi}$ and $\mathrm{Ni}$ layers, and on an electric dipole generated in the Bi bilayer on (111) twist GB. The stability of Bi films on low energy $\Sigma$ 3(111) and high energy $\Sigma 5(120)$ transition metal GBs is studied in [13].

Fig. 6(A) shows the GB free energy of Bi films at a Ni $\Sigma 5(120) \mathrm{GB}$, where the Bi bilayer complexion is stable for $-0.37 \mathrm{eV}<\Delta \mu_{B i}<0 \mathrm{eV}$. Results of stabilities of other segragate and host couples are shown in Tab. 1. A similar calculation on the $\mathrm{Cu} \Sigma 5(120)$ GB finds the bilayer stable only over $-0.067 \mathrm{eV}<\Delta \mu_{B i}<0 \mathrm{eV}$, which is consistent with the experimental observations $[6,45]$ that Bi bilayer complexions are more frequent in larger areas in $\mathrm{Ni}$ than $\mathrm{Cu}$. The vibrational free energy only increases the enthalpies of formation by around $0.01 \mathrm{eV} / \AA^{2}$ and does not alter the film stability at $T=1000 \mathrm{~K}$ [13]. DFT calculations show the cohesive energies at interfaces decreased by around $95 \%$ due to the formation of Bi bi- 

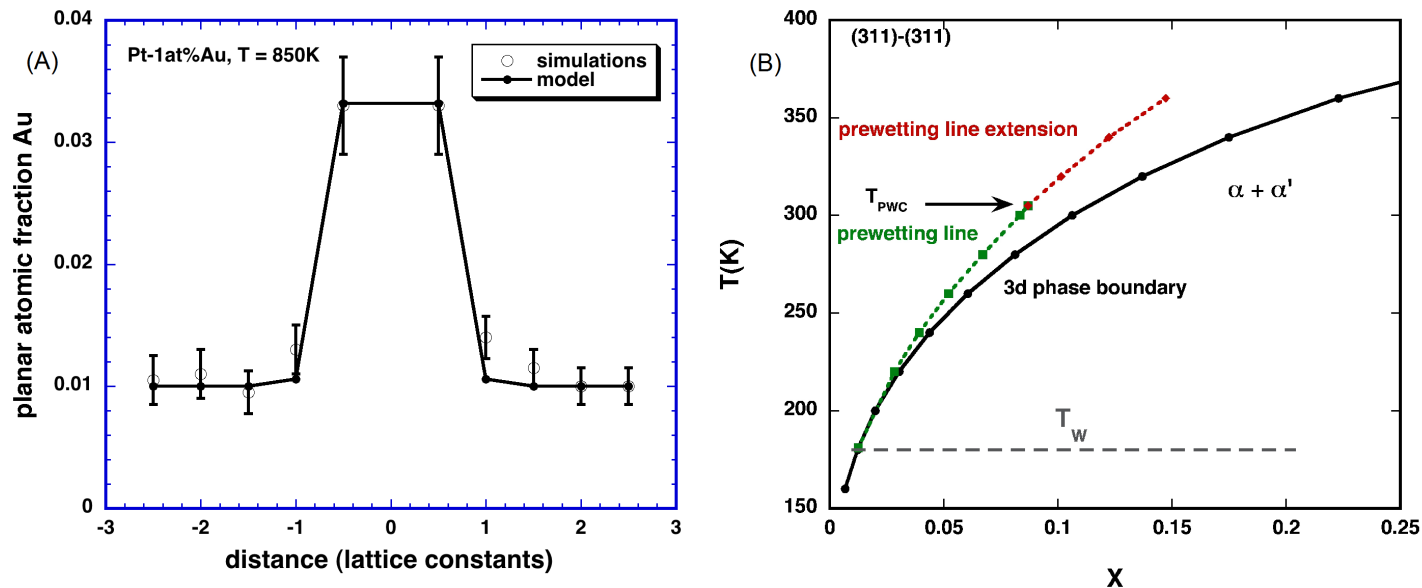

Figure 5: (Color online) (A) GB composition of Pt-1 at \% Au (100) $43.6^{\circ} \mathrm{GB}$ at $850 \mathrm{~K}$ Model predicted by model of Wynblatt et al [48] and MC simulation [49]. Error bars on simulation results represent one standard deviation. (B) Portion of the three-dimensional phase diagram for the model alloy with certain parameters, showing superposition of the prewetting line and its extension for a FCC (311)-(311) GB generated by model of Wynblatt et al [7]. (A) is reprinted from [48] with permission and (B) is reprinted from [7] with permission.

laver [12. 131.

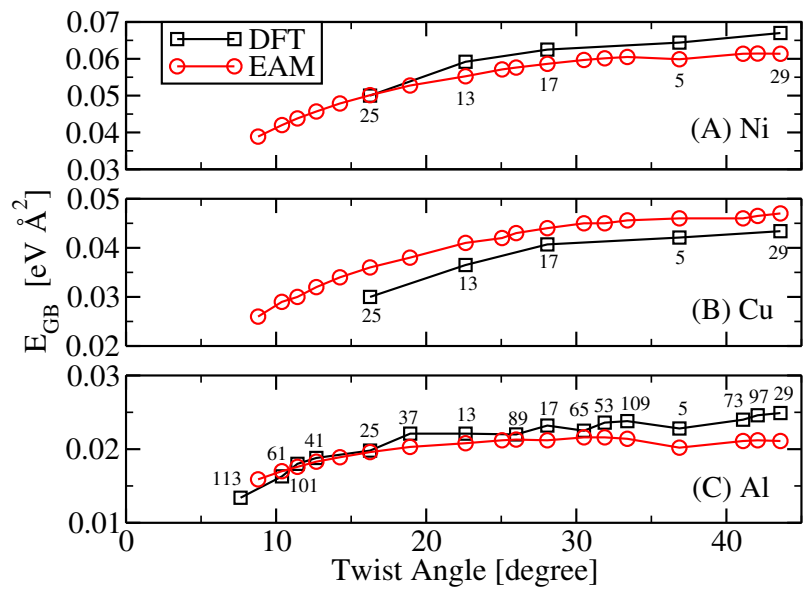

Figure 7: (Color online) Grain boundary energies calculated by DFT compared with the embedded atom method (EAM) for (A) Ni [13], (B) $\mathrm{Cu}$ and (C) Al. The EAM data is taken from [60]. Numbers shown in the figure are the values of those (100) CSL GBs. For both metals, the results show similar trends and differ mostly by less than $0.005 \mathrm{eV} / \AA^{2}$.

Fig. 6(B) and (C) show the charge density differences of bilayer and four-layer Bi complexions at $\mathrm{Ni} \Sigma 5(120)$

\begin{tabular}{|c|c|c|c|c|}
\hline seg/host & $\Delta \mu_{0 \rightarrow 1}$ & $\Delta \mu_{1 \rightarrow 2}$ & $\Delta \mu_{2 \rightarrow 4}$ & $\Delta \mu_{4 \rightarrow \infty}$ \\
\hline $\mathrm{Bi} / \mathrm{Co}$ & \multicolumn{4}{|c|}{$\Delta \mu_{0 \rightarrow \infty}=0$} \\
\hline $\mathrm{Bi} / \mathrm{Ni}$ & \multicolumn{3}{|c|}{$\Delta \mu_{0 \rightarrow 2}=-0.37$} & \multicolumn{2}{|c|}{$\Delta \mu_{2 \rightarrow \infty}=0$} \\
\hline $\mathrm{Bi} / \mathrm{Cu}$ & \multicolumn{2}{|c|}{$\Delta \mu_{0 \rightarrow 2}=-0.067$} & \multicolumn{2}{|c|}{$\Delta \mu_{2 \rightarrow \infty}=0$} \\
\hline $\mathrm{Bi} / \mathrm{Pd}$ & -1.43 & -0.52 & -0.07 & 0 \\
\hline $\mathrm{Bi} / \mathrm{Pt}$ & -0.98 & -0.25 & -0.08 & 0 \\
\hline $\mathrm{As} / \mathrm{Ni}$ & \multicolumn{2}{|c|}{$\Delta \mu_{0 \rightarrow 2}=-0.88$} & -0.02 & 0 \\
\hline $\mathrm{Sb} / \mathrm{Ni}$ & -1.03 & -0.38 & $\Delta \mu_{2 \rightarrow \infty}=0$ \\
\hline
\end{tabular}

Table 1: DFT calculated relative chemical potentials $\Delta \mu$ of the transition points of segregate (seg) on the $\Sigma 5(120)$ GB of host. For example, at $\Delta \mu<\Delta \mu_{0 \rightarrow 1}$ bare GB is stable, at $\Delta \mu_{0 \rightarrow 1}<\Delta \mu<\Delta \mu_{1 \rightarrow 2}$ monolayer complexion is stable, etc. Units are in $\mathrm{eV}$.

GB. The charge density difference $\Delta \rho$ is defined as

$$
\Delta \rho=\rho(\mathrm{Bi}-\mathrm{Ni} ; \mathrm{SCF})-\rho(\mathrm{Ni} ; \mathrm{SCF})-\rho(\mathrm{Bi} ; \text { atomic }),
$$

where $\rho(\mathrm{Bi}-\mathrm{Ni} ; \mathrm{SCF})$ is the self-consistant DFT calculated charge density of Bi segregated $\mathrm{Ni} \mathrm{GB}, \rho(\mathrm{Ni} ; \mathrm{SCF})$ is the self-consistant DFT calculated charge density of $\mathrm{Ni}$ GB with same geometry of Bi segregated Ni GB but Bi atoms removed, and $\rho(\mathrm{Bi} ;$ atomic $)$ is the DFT calculated atomic charge density of all $\mathrm{Bi}$ atoms. Fig. 6(B) and (C) indicate the strong interaction between $\mathrm{Ni}$ atoms at $\mathrm{GB}$ and the adjacent $\mathrm{Bi}$ layer, the strong interaction within the middle Bi bilayer of the four-layer complexion, and 


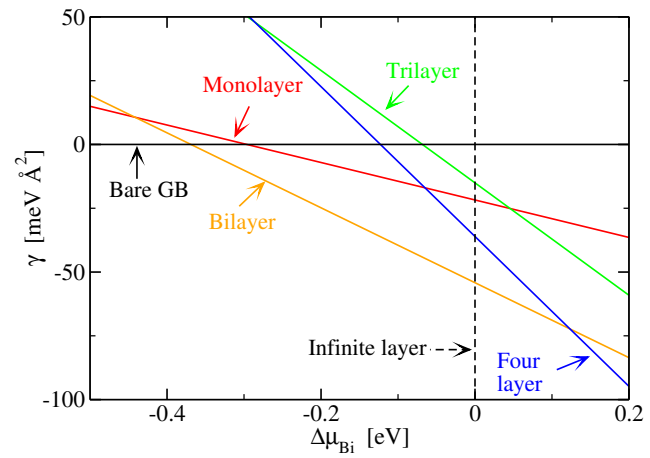

(A)

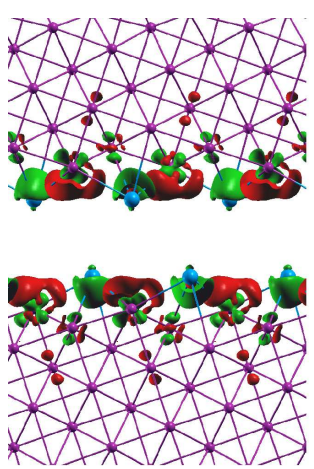

(B)
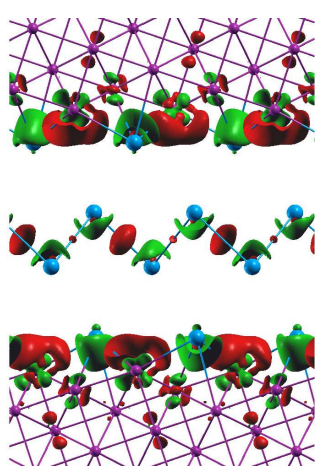

(C)

Figure 6: (Color online) (A) GB free energy of Bi films at Ni $\Sigma 5(120)$ GBs [13]. The black solid lines stand for bare GBs while the black dashed lines stand for infinite bulk-like Bi films. Other lines are for different Bi films. (B) Electronic charge density difference $\Delta \rho$ plot of bilayer Bi films at $(1 \times 1)$ cell of Ni $5(120) \mathrm{GB}$. Ni atoms are shown in purple, Bi in blue. The charge contour surfaces are plotted for $\Delta \rho=0.029 e V / \AA^{3}$. Red for charge excess and green for charge deficient. (C) Electronic charge density difference plot of four-layer Bi films at (1×1) cell of Ni 5(120) GB, same definition as in (B).

the weak interaction between the $\mathrm{Bi}$ layer next to $\mathrm{Ni}$ and other Bi layer(s). The difference between $\mathrm{Bi}$ at $\mathrm{Co}, \mathrm{Ni}$ and $\mathrm{Cu}$ GBs is analyzed based on localization of orbitals and magnetization with crystal orbital Hamilton populations calculations [13].

By exploiting the weak Bi interlayer interaction in bilayer complexions, a thermodynamics model was proposed that can be used to predict Bi bilayer stability on various Ni GBs with relatively simple surface calculations [13]. Approximate energies for many bare GBs can be obtained from embedded atom method (EAM) calculations [61, 62, 60, 63, 64]. The difference between DFT and EAM calculated (100) coincidence site lattice (CSL) twist GBs are mostly within $0.005 \mathrm{eV} / \AA^{2}$ as shown in Fig. 7. Based on the EAM bare GB energies, the model predicts that $\mathrm{Bi}$ bilayer are unstable on all Ni(111) CSL twist GBs, but are stable on (100) CSL twist GBs for rotation angles between 10 and 45 degrees.

\subsection{Molecular dynamics and Monte Carlo simulations of complexion transitions}

Complexion transitions can dramatically change material properties [65]. However, since high-temperature GB observation is difficult, no direct complexion transition has been observed so far. Computer simulation of complexion transitions involves the evolution of $\sim 10^{4}$ atoms in time scale of tens of nanoseconds which is infeasible with DFT calculations but feasible with less accurate empirical potential methods.

Recently, complexion transitions in pure $\mathrm{Cu} \Sigma 5(120)$ and $\Sigma 5(310)$ were demonstrated with empirical potential methods based molecular dynamics simulation [14], as shown in Fig. 8. The authors utilized a new methodology that allows variations in atomic density inside the GB by terminating GB with an open surface at one end and a wall of fixed atoms at the other end. The coexistence of two complexions, kites and split kites, is demonstrated (Fig. 8(A)) at $800 \mathrm{~K}$ on a $\Sigma 5(310) \mathrm{GB}$. A line defect with an atomic scale cross-section separates the two complexions. When terminating the GB with two free surfaces, the whole GB transforms into the split kites at $800 \mathrm{~K}$. On the $\Sigma 5(120) \mathrm{GB}$, the reversible complexion transition between split kites at $1000 \mathrm{~K}$ and filled kites at $1100 \mathrm{~K}$ was shown. Similar simulation methods with $\mathrm{MC}$ and MD [16] directly shows that the distinct breaks in the slopes observed in an Arrhenius plot of $\mathrm{Ag}$ and $\mathrm{Au}$ diffusion in $\mathrm{Cu} \Sigma 5(310) \mathrm{GB}[66,67]$ are due to a $\mathrm{Cu} \mathrm{GB}$ complexion transition. The Ag segregation-induced phase transition in $\mathrm{Cu} \Sigma 5(120) \mathrm{GB}$ is also demonstrated [15], as shown in Fig. 8. 


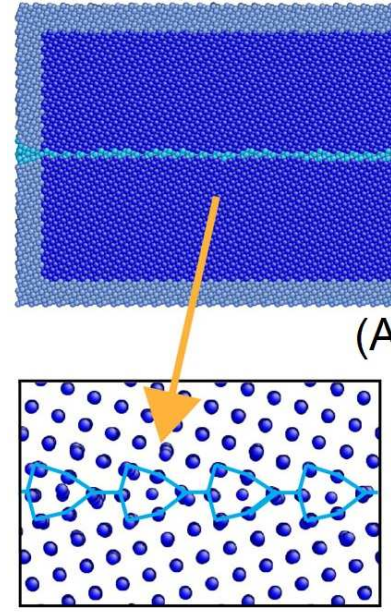

(B) Kites
(A)

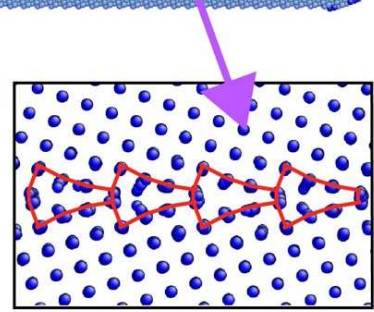

(C) Split kites

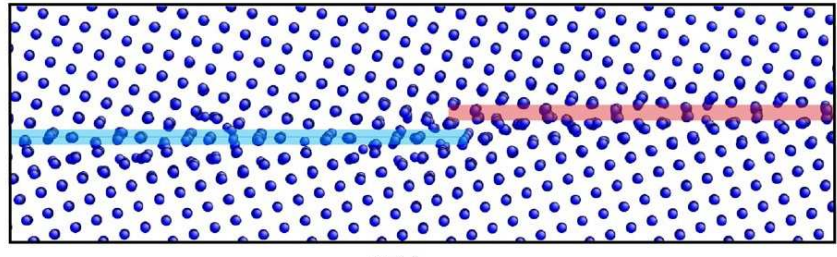

(D)

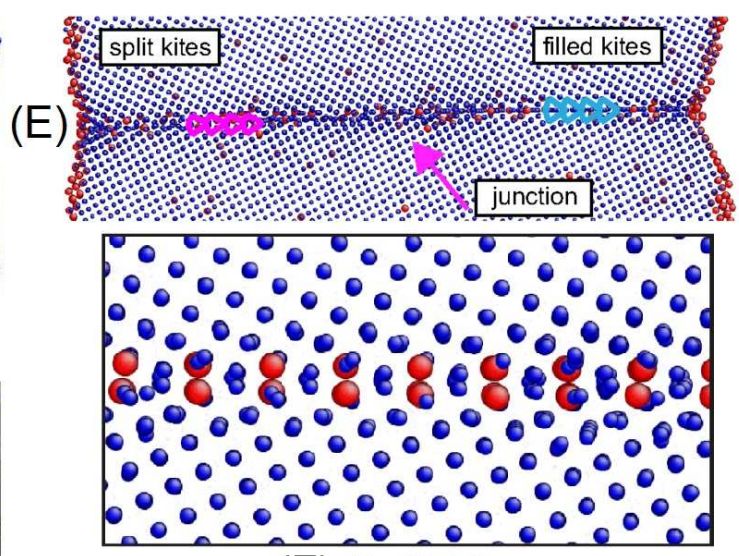

(F) Split kites

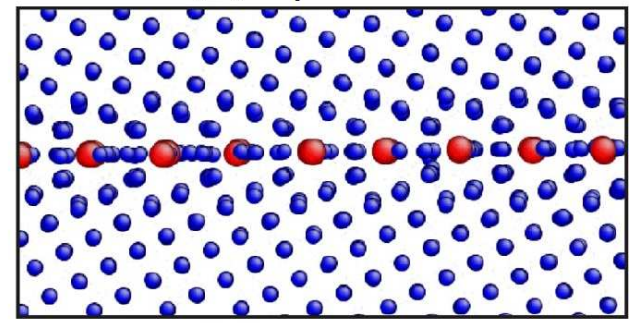

(G) Filled kites

Figure 8: (Color online) Complexion transformation of pure $\mathrm{Cu} \Sigma 5(310) \mathrm{GB}$ and $\mathrm{Ag}$ segregated $\mathrm{Cu} \Sigma 5(120) \mathrm{GB}$. (A) Coexistance of kites complexion and split kites complexion with the geometry of a wall of fixed atoms at left and an open surface at right. $\mathrm{Cu}(\mathrm{B})$ kites and (C) split kites complexion at $\mathrm{Cu} \Sigma 5(310)$ and (D) their 1D-phase boundary separation accompanied by a step. (E) Coexistance of spit kites complexion and filled kites complexion with the geometry of open surfaces at both left and right of Ag segregated $\mathrm{Cu} \Sigma 5(120) \mathrm{GB}$. Ag segregated $\mathrm{Cu}(\mathrm{F})$ split kites and (G) filled kites complexion at $\mathrm{Cu} \Sigma 5(120)$. (A-D) is reprinted from [14] with permission. (E-G) is reprinted from [15] with permission.

\section{Outstanding challenges}

The long-term goal is to understand and predict complexions and to tailor material properties by complexion engineering. The first crucial step is to understand the structures of complexions and the conditions that stabilize them. For the surface complexion, an atomic-resolved experiment of $\mathrm{Bi}$ on $\mathrm{Ni}(111)$ is warranted to end the debate over the Bi surface structures and stability mechanism [32, 20]. For the GB complexion, although the bilayer complexion is observed, the exact complexion structures and their relation with complexion properties are still unknown. Structural modeling based on direct HAADF-STEM observation and combined with DFT verification is needed. Since a strong effect of additives on the formation of IGF has been demonstrated [41], the effect of additives on $\mathrm{Bi}$ (sub)nanometer complexions is also an attractive question. Complexion transitions like prewetting/premelting, faceting, roughening also remain to be observed and analyzed [58].

Analytical models have the advantage of representing the whole space of GB configurations, but lack accurate total energies and entropies, while DFT calculations can accurately deal only with small cell sizes. It is thus desirable to feed DFT calculated interaction parameters into analytical models. MC and MD simulations using empirical potentials successfully applied to $\mathrm{Cu}$ and $\mathrm{Ag} / \mathrm{Cu}$ can in principle be used to study $\mathrm{Bi} / \mathrm{Ni}$ and $\mathrm{Bi} / \mathrm{Cu}$ complexion transitions, but only if accurate interaction potentials can be obtained. One approach is to develop DFT-based machine learning models, which are capable of learning 
atomic interactions from small-cell DFT calculations and generalizing to large cell simulations [68].

\section{Acknowledgements}

The authors thank Jian Luo, Zhiyang Yu, Jeffrey Rickman, Anthony Rollett, Gregory Rohrer and Martin Harmer for helpful discussion. Financial support from the ONR-MURI under Grant No. N00014-11-1-0678 and from DOE under Grant No. DE-SC0014506 is gratefully acknowledged.

[1] L. Felton, C. Raeder, and D. Knorr, JOM 45, 28 (1993), ISSN 1047-4838.

[2] H. Zhu, J. Hu, Y. Zhang, and Y. Fei, in Advanced Tribology, edited by J. Luo, Y. Meng, T. Shao, and Q. Zhao (Springer Berlin Heidelberg, 2010), pp. 878-879.

[3] N. Marie, K. Wolski, and M. Biscondi, Journal of Nuclear Materials 296, 282 (2001), 4th Int. Workshop on Spallation Materials Technology.

[4] B. Straumal and B. Baretzky, Interface Science 12, 147 (2004).

[5] K. Wolski and V. Laporte, Materials Science and Engineering: A 495, 138 (2008), fifth International Conference on High Temperature Capillarity HTC2007, Alicante, Spain.

[6] J. Luo, H. Cheng, K. M. Asl, C. Kiely, and M. Harmer, Science 333, 1730 (2011).

[7] P. Wynblatt and D. Chatain, Materials Science and Engineering: A 495, 119 (2008), fifth International Conference on High Temperature Capillarity HTC2007, Alicante, Spain.

[8] J. W. Cahn, The Journal of Chemical Physics 66, 3667 (1977).

[9] R. Kobayashi, J. A. Warren, and W. C. Carter, Physica D: Nonlinear Phenomena 140, 141 (2000).

[10] J. A. Warren, R. Kobayashi, A. E. Lobkovsky, and W. C. Carter, Acta Materialia 51, 6035 (2003).
[11] P. R. Cantwell, M. Tang, S. J. Dillon, J. Luo, G. S. Rohrer, and M. P. Harmer, Acta Materialia 62, 1 (2014).

[12] J. Kang, G. C. Glatzmaier, and S.-H. Wei, Phys. Rev. Lett. 111, 055502 (2013).

[13] Q. Gao and M. Widom, Phys. Rev. B 90, 144102 (2014).

[14] T. Frolov, D. L. Olmsted, M. Asta, and Y. Mishin, Nat Commun 4 (2012).

[15] T. Frolov, M. Asta, and Y. Mishin, Phys. Rev. B 92, 020103 (2015).

[16] T. Frolov, S. V. Divinski, M. Asta, and Y. Mishin, Phys. Rev. Lett. 110, 255502 (2013).

[17] G. Duscher, M. F. Chisholm, U. Alber, and M. Ruhle, Nat. Mater. 3, 621 (2004).

[18] R. Schweinfest, A. Paxton, and M. W. Finnis, Nature 432, 1008 (2004).

[19] P. Hofmann, Prog. in Surf. Sci. 81, 191 (2006).

[20] Q. Gao and M. Widom, Phys. Rev. B 88, 155416 (2013).

[21] F. Delamare and G. Rhead, Surface Science 35, 172 (1973).

[22] W. D. Clendening and C. T. Campbell, The Journal of Chemical Physics 90, 6656 (1989).

[23] B. Blum and H. Ascolani, Surface Science 482.485, Part 2, 946 (2001).

[24] D. Kaminski, P. Poodt, E. Aret, N. Radenovic, and E. Vlieg, Surface Science 575, 233 (2005).

[25] J. Monchoux, D. Chatain, and P. Wynblatt, Surface Science 600, 1265 (2006).

[26] P. Wynblatt, D. Chatain, and A. Ranguis, Surface Science 601, 1623 (2007).

[27] Y. Girard, C. Chacon, G. de Abreu, J. Lagoute, V. Repain, and S. Rousset, Surface Science 617, 118 (2013). 
[28] R. van Gastel, D. Kaminski, E. Vlieg, and B. Poelsema, Phys. Rev. B 89, 075431 (2014).

[29] T. Nagao, J. T. Sadowski, M. Saito, S. Yaginuma, Y. Fujikawa, T. Kogure, T. Ohno, Y. Hasegawa, S. Hasegawa, and T. Sakurai, Phys. Rev. Lett. 93, 105501 (2004).

[30] V. Fourneé, H. R. Sharma, M. Shimoda, A. P. Tsai, A. R. R. B. Unal, T. A. Lograsso, and P. A. Thiel, Phys. Rev. Lett. 95, 155504 (2005).

[31] S. Yaginumaa, T. Nagaoa, J. Sadowski, M. Saito, K. Nagaoka, Y. Fujikawa, T. Sakurai, and T. Nakayama, Surf. Sci. 601, 3593 (2007).

[32] T. R. J. Bollmann, R. van Gastel, H. J. W. Zandvliet, and B. Poelsema, Phys. Rev. Lett. 107, 176102 (2011).

[33] J. P. Perdew, K. Burke, and M. Ernzerhof, Phys. Rev. Lett. 77, 3865 (1996).

[34] T. Hirahara, G. Bihlmayer, Y. Sakamoto, M. Yamada, H. Miyazaki, S.-i. Kimura, S. Blügel, and S. Hasegawa, Phys. Rev. Lett. 107, 166801 (2011).

[35] F. Yang, L. Miao, Z. F. Wang, M.-Y. Yao, F. Zhu, Y. R. Song, M.-X. Wang, J.-P. Xu, A. V. Fedorov, Z. Sun, et al., Phys. Rev. Lett. 109, 016801 (2012).

[36] X.-X. Gong, H.-X. Zhou, P.-C. Xu, D. Yue, K. Zhu, X.-F. Jin, H. Tian, G.-J. Zhao, and T.-Y. Chen, Chinese Physics Letters 32, 067402 (2015).

[37] L.-S. Chang, E. Rabkin, B. Straumal, B. Baretzky, and W. Gust, Acta Materialia 47, 4041 (1999).

[38] B. Straumal, S. Polyakov, E. Bischoff, W. Gust, and B. Baretzky, Acta Materialia 53, 247 (2005).

[39] K. Wolski, V. Laporte, N. Marié, and M. Biscondi, Interface Science 9, 183 (2001).

[40] E. Pereiro-López, W. Ludwig, D. Bellet, P. Cloetens, and C. Lemaignan, Phys. Rev. Lett. 95, 215501 (2005).

[41] K. M. Asl and J. Luo, Acta Materialia 60, 149 (2012).
[42] N. Marié, K. Wolski, and M. Biscondi, Scripta Materialia 43, 943 (2000).

[43] J. Schölhammer, B. Baretzky, W. Gust, E. Mittemeijer, and B. Straumal, Interface Science 9, 43 (2001).

[44] L.-S. Chang and K.-B. Huang, Scripta Materialia 51, 551 (2004).

[45] A. Kundu, K. M. Asl, J. Luo, and M. P. Harmer, Scripta Mater. 68, 146 (2013).

[46] B. Straumal, O. Kogtenkova, and P. Zięba, Acta Materialia 56, 925 (2008).

[47] Y. Zhang and J. Luo, Scripta Materialia 88, 45 (2014).

[48] P. Wynblatt and Z. Shi, Journal of Materials Science 40, 2765 (2005).

[49] D. Udler and D. N. Seidman, physica status solidi (b) 172, 267 (1992).

[50] D. McLean (1957).

[51] Y. Lee and H. Aaronson, Acta Metallurgica 28, 539 (1980).

[52] Y. Lee and H. Aaronson, Surface Science 95, 227 (1980).

[53] D. A. Steigerwald, S. J. Miller, and P. Wynblatt, Surface Science 155, 79 (1985).

[54] P. Wynblatt and R. Ku, Surface Science 65, 511 (1977).

[55] J. Friedel, Advances in Physics 3, 446 (1954).

[56] J. Rickman, H. Chan, M. Harmer, and J. Luo, Surface Science 618, 88 (2013).

[57] M. Tang, W. C. Carter, and R. M. Cannon, Phys. Rev. Lett. 97, 075502 (2006).

[58] P. R. Cantwell, M. Tang, S. J. Dillon, J. Luo, G. S. Rohrer, and M. P. Harmer, Acta Materialia 62, 1 (2014).

[59] J. Luo, Applied Physics Letters 95, 071911 (2009). 
[60] E. A. Holm, D. L. Olmsted, and S. M. Foiles, Scripta Materialia 63, 905 (2010).

[61] D. L. Olmsted, S. M. Foiles, and E. A. Holm, Acta Materialia 57, 3694 (2009).

[62] M. D. Sangid, H. Sehitoglu, H. J. Maier, and T. Niendorf, Materials Science and Engineering: A 527, 7115 (2010).

[63] G. S. Rohrer, E. A. Holm, A. D. Rollett, S. M. Foiles, J. Li, and D. L. Olmsted, Acta Materialia 58, 5063 (2010).

[64] S. Ratanaphan, D. L. Olmsted, V. V. Bulatov, E. A. Holm, A. D. Rollett, and G. S. Rohrer, Acta Materialia 88, 346 (2015).

[65] Y. Mishin, M. Asta, and J. Li, Acta Materialia 58, 1117 (2010).

[66] S. V. Divinski, H. Edelhoff, and S. Prokofjev, Phys. Rev. B 85, 144104 (2012).

[67] E. Budke, T. Surholt, S. Prokofjev, L. Shvindlerman, and C. Herzig, Acta Materialia 47, 385 (1999).

[68] J. Behler and M. Parrinello, Phys. Rev. Lett. 98, 146401 (2007). 